08,04

\title{
Орторомбические центры редкоземельных $S$-ионов в кристаллах лютеций-алюминиевого граната
}

\author{
(C) В.А. Важенин ${ }^{1}$, А.П. Потапов ${ }^{1}$, Г.Р. Асатрян ${ }^{2}$, А.Г. Петросян ${ }^{3}$, \\ А.В. Фокин ${ }^{1}$, М.Ю. Артёмов ${ }^{1}$ \\ ${ }^{1}$ Институт естественных наук Уральского фредерального университета, \\ Екатеринбург, Россия \\ ${ }^{2}$ Физико-технический институт им. А.Ф. Иоффре РАН, \\ Санкт-Петербург, Россия \\ ${ }^{3}$ Институт физических исследований НАН Армении, \\ Аштарак, Армения \\ E-mail: Vladimir.Vazhenin@urfu.ru
}

(Поступила в Редакцию 13 декабря 2016 г.)

Исследован электронный парамагнитный резонанс центров $\mathrm{Eu}^{2+}$ и $\mathrm{Gd}^{3+}$ в монокристаллах $\mathrm{Lu}_{3} \mathrm{Al}_{5} \mathrm{O}_{12}$. Определены параметры ромбического спинового гамильтониана этих центров.

Работа выполнена в рамках государственного задания Минобрнауки РФ для Уральского федерального университета, а также поддержана РФФИ (грант № 15-52-05040 Арм_а) и Госкомитетом по науке Армении (грант 15RF-003).

Измерения проведены на спектрометре Центра коллективного пользования „Современные нанотехнологии“ Уральского федерального университета.

DOI: 10.21883/FTT.2017.07.44596.442

\section{1. Введение}

Эмпирическая суперпозиционная модель для основного состояния парамагнитного иона в кристалле, предложенная авторами $[1,2]$, и ее версия для редкоземельных $S$-ионов $[3,4]$ были разработаны с целью связать величины параметров начального расщепления основного состояния (тонкой структуры спектра ЭПР) с координатами ближайшего окружения и, следовательно, оценить релаксацию этого окружения вследствие замены иона. В работах [5-7] показано, что наиболее сильно на искажения окружения откликаются компоненты тензора тонкой структуры второго ранга.

У парамагнитных центров $S$-ионов с осевой симметрией $\left(C_{3}, C_{4}, S_{4}, C_{6}\right)$ указанный тензор имеет одну компоненту, что позволяет в версии [3] лишь оценить внутренний параметр модели, учитывающий эффекты обмена парамагнитный ион-лиганд, предполагая при этом отсутствие релаксации окружения.

В случае центров орторомбической симметрии (например, ионы $\mathrm{Eu}^{2+}$ и $\mathrm{Gd}^{3+}$ в кристаллах со структурой граната [8-12]) тензор тонкой структуры второго ранга имеет две компоненты, что дает принципиальную возможность сделать некоторые заключения о релаксации лигандного окружения. Можно ожидать, что такой анализ будет успешным при наличии параметров тонкой структуры $S$-ионов в ряду кристаллов со структурой граната. В настоящее время ромбические центры $\mathrm{Gd}^{3+}$ исследованы в $\mathrm{Y}_{3} \mathrm{Al}_{5} \mathrm{O}_{12} \quad[8,10]$ и $\mathrm{Y}_{3} \mathrm{Ga}_{5} \mathrm{O}_{12} \quad[8,9]$, а центры $\mathrm{Eu}^{2+}$ — только в $\mathrm{Y}_{3} \mathrm{Al}_{5} \mathrm{O}_{12}[11,12]$. В данной работе содержатся результа- ты ЭПР-исследования центров $\mathrm{Eu}^{2+}$ и $\mathrm{Gd}^{3+}$ еще в одном гранате $\left(\mathrm{Lu}_{3} \mathrm{Al}_{5} \mathrm{O}_{12}\right)$.

\section{2. Образцы и методика измерений}

Монокристаллы $\mathrm{Lu}_{3} \mathrm{Al}_{5} \mathrm{O}_{12}$ были выращены вертикальным методом Бриджмена в молибденовом тигле с использованием чистых (99.99\%) исходных оксидов $\mathrm{Lu}_{2} \mathrm{O}_{3}, \mathrm{Eu}_{2} \mathrm{O}_{3}$ и $\mathrm{Al}_{2} \mathrm{O}_{3}$. Для стабилизации высоких концентраций $\mathrm{Eu}^{2+}$ в расплав вводилась дополнительная четырехвалентная примесь в виде оксида $\mathrm{SiO}_{2}$. Концентрации примесей составляли 0.1 at.\% для европия и 0.1 at.\% для кремния.

Измерения ЭПР-спектров образцов проводились на спектрометре трехсантиметрового диапазона EMX Plus Bruker в интервале температур 120-300 К. На неориентированных кристаллах измерять угловые зависимости спектра в определенных плоскостях было затруднительно. Ориентация образца в резонаторе спектрометра в случаях: $\mathbf{B}\left\|\mathbf{C}_{2}, \quad \mathbf{B}\right\| \mathbf{C}_{3}, \quad \mathbf{B} \| \mathbf{C}_{4} \quad(B$-индукция магнитного поля, $C_{i}$ - оси кристалла) осуществлялась с помощью штатного одноосного автоматического гониометра. Для реализации вращения в вертикальной плоскости кристалл помещался в тефлоновый бочонок, расположенный на штанге гониометра и обеспечивающий необходимое вращение.

Начальное ориентирование образца производилось по спектру тригональных центров $\mathrm{Mo}^{3+}$, исследованных авторами [13], финальное — с учетом угловых зависимостей положений переходов ромбических центров $\mathrm{Gd}^{3+}$ (рис. 1). Ионы молибдена и гадолиния в качестве 


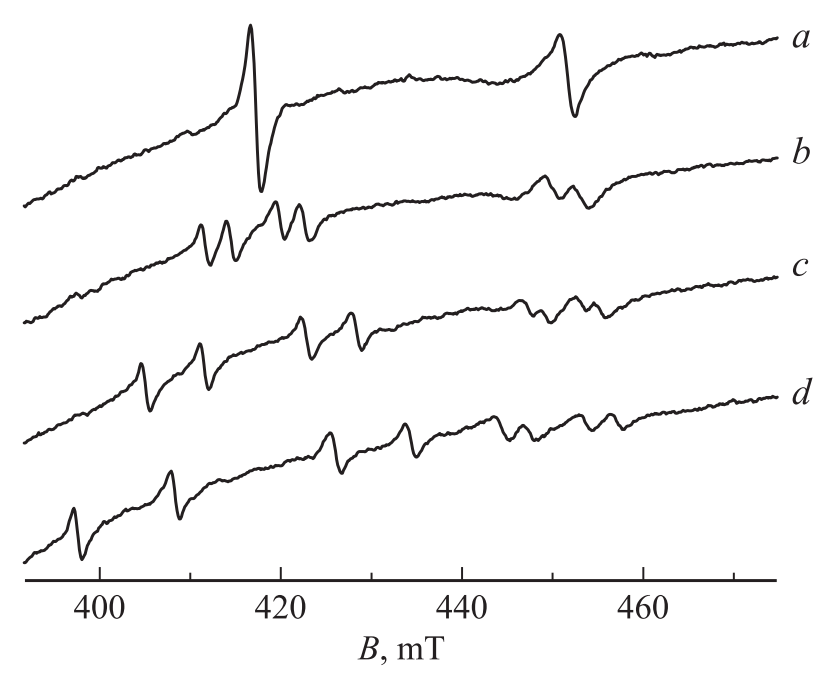

Рис. 1. Спектр двух переходов четырех центров $\mathrm{Gd}^{3+}$ (эквивалентных в ориентации $\left.\mathbf{B} \| \mathbf{C}_{4}\right)$ при отклонении от $\mathbf{B} \| \mathbf{C}_{4}$, $300 \mathrm{~K}$, рабочая частота $9874 \mathrm{MHz} . a-\mathbf{B} \| \mathbf{C}_{4}, b-1^{\circ}$ от $\mathbf{B} \| \mathbf{C}_{4}, c-2^{\circ}$ от $\mathbf{B} \| \mathbf{C}_{4}, d-3^{\circ}$ от $\mathbf{B} \| \mathbf{C}_{4}$.

неконтролируемой примеси присутствуют в кристалле и дают интенсивные спектры.

\section{3. Результаты измерений}

На рис. 2,3 приведены ЭПР-спектры (производная спектра поглощения) при $\mathbf{B} \| \mathbf{C}_{2}$ и $\mathbf{B} \| \mathbf{C}_{4}$, демонстрирующие переходы центров $\mathrm{Eu}^{2+}, \mathrm{Gd}^{3+}$ и $\mathrm{Mo}^{3+}$ в $\mathrm{Lu}_{3} \mathrm{Al}_{5} \mathrm{O}_{12}$. Видно, что, как и в $\mathrm{Y}_{3} \mathrm{Al}_{5} \mathrm{O}_{12}[11,12]$, низкополевые переходы центров $\mathrm{Eu}^{2+}$ в $\mathrm{Lu}_{3} \mathrm{Al}_{5} \mathrm{O}_{12}$ имеют частично разрешенную сверхтонкую структуру (СТС) (ядерные спины изотопов ${ }^{151} \mathrm{Eu},{ }^{153} \mathrm{Eu}$ равны $5 / 2$, естественная распространенность 48 и $52 \%$ соответственно), тогда как высокополевые представляют собой широкие бесструктурные линии. В связи с этим измерение положений сверхтонких компонент на большинстве переходов было невозможно. Поэтому для определения параметров начального расщепления ионов $\mathrm{Eu}^{2+}$ нами использовались положения „центров“ СТС (как правило, несимметричной) и центров бесструктурных линий, что привело к большим погрешностям в $b_{n m}\left(b_{n m}-\right.$ параметры спинового гамильтониана в определении [14]).

Положения узких сигналов четных изотопов $\mathrm{Gd}^{3+}$ (рис. 2,3 ) измерялись намного точнее, что, естественно, позволило уменьшить ошибки в определении параметров тонкой структуры. При $\mathbf{B} \| \mathbf{C}_{3}$ и $120 \mathrm{~K}$ сигнал параллельного тригонального центра $\mathrm{Mo}^{3+}$ (переход $1 / 2--1 / 2$ ) демонстрировал характерную внутреннюю структуру (пять плохо разрешенных компонент), обусловленную, согласно [13], суперсверхтонким взаимодействием с шестью ближайшими ядрами ${ }^{175} \mathrm{Lu}$.

Редкоземельные ионы $\mathrm{Gd}^{3+}$ и $\mathrm{Eu}^{2+}$ в структуре граната (пространственная группа Ia $3 d\left(O_{h}^{10}\right)$ ) занимают позицию иона $\mathrm{Lu}^{3+}$ с симметрией $D_{2}$ и образуют шесть эквивалентных, но различно ориентированных центров. В ориентации B $\| \mathbf{C}_{2}$ четыре центра из шести эквивалентны $\left(\theta=60^{\circ}, \varphi=35.3^{\circ}\right)$ и их спектры суммируются (рис. 2 расчетные положения и интенсивности центра $\mathrm{Eu}^{2+}$ показаны пунктирными отрезками), а оставшиеся два центра являются одиночными с $\theta=0^{\circ}$ и $\theta=90^{\circ}$, $\varphi=90^{\circ}$. Следует заметить, что определение того, какой из наблюдаемых спектров является параллельным, а какой - перпендикулярным, представляет собой весьма нетривиальную задачу. При $\mathbf{B} \| \mathbf{C}_{4}$ (рис. 3) эквивалентны два центра с $\theta=90^{\circ}, \varphi=0^{\circ}$ и четыре с $\theta=45^{\circ}$, $\varphi=90^{\circ}$.

Оси $Z$ и $Y$ используемой нами системы координат совпадают с осями второго порядка группы симметрии позиции $D_{2}$, параллельными осям $C_{2}$ кристалла, ось $X$ параллельна третьей оси $C_{2}$ группы $D_{2}$ и оси $C_{4}$ кристалла. Используя результаты работ $[11,12]$ и предполагая близость параметров спинового гамильтониана центров $\mathrm{Gd}^{3+}$ и $\mathrm{Eu}^{2+}$ в двух гранатах $\left(\mathrm{Y}_{3} \mathrm{Al}_{5} \mathrm{O}_{12}\right.$ и $\left.\mathrm{Lu}_{3} \mathrm{Al}_{5} \mathrm{O}_{12}\right)$, мы смогли идентифицировать переходы указанных центров. Пришлось перебрать несколько вариантов, поскольку близость этих параметров в двух гранатах оказалась весьма условной (см. далее). Минимизация среднеквадратичных отклонений расчетных частот от экспериментальных при двух температурах привела к параметрам спинового гамильтониана (в определении [14]), представленным в табл. 1. Параметры шестого ранга оказались меньше погрешности измерений и здесь не приводятся. Естественно, что в процедуре минимизации сверхтонкое и квадрупольное, а также ядерное зеемановское взаимодействие не учитывались. Обращает на себя внимание бо́льшая величина (по сравнению с таковой для центров $\mathrm{Gd}^{3+}$ ) среднеквадратичного отклонения расчетных частот от экспериментальных для центров $\mathrm{Eu}^{2+}$. Этот факт, как и бо́льшая погрешность $b_{n m}$, обусловлен неточностью определения положений переходов центров

Таблица 1. Параметры спинового гамильтониана $b_{n m}$ и среднеквадратичные отклонения расчетных частот от экспериментальных $F(N)$ ( $N$ - число использованных экспериментальных точек) для ромбических центров $\mathrm{Eu}^{2+}$ (в двух системах координат) и $\mathrm{Gd}^{3+}$ в $\mathrm{Lu}_{3} \mathrm{Al}_{5} \mathrm{O}_{12}$.

\begin{tabular}{c|c|c|c|c|c}
\hline \multirow{2}{*}{ Параметры } & \multicolumn{2}{|c|}{$\mathrm{Gd}^{3+}$} & \multicolumn{3}{c}{$\mathrm{Eu}^{2+}$} \\
\cline { 2 - 6 } & \multicolumn{2}{|c|}{$\mathbf{Z} \| \mathbf{C}_{2}(1)$} & $\mathbf{Z} \| \mathbf{C}_{2}(2)$ & \multicolumn{2}{|c}{$\mathbf{Z} \| \mathbf{C}_{2}(1)$} \\
\cline { 2 - 6 } & $300 \mathrm{~K}$ & $120 \mathrm{~K}$ & $300 \mathrm{~K}$ & $300 \mathrm{~K}$ & $120 \mathrm{~K}$ \\
\hline$g$ & \multicolumn{2}{|c|}{1.991} & \multicolumn{3}{|c}{1.9905} \\
$b_{20} \mathrm{MHz}$ & $1750(2)$ & $1754(3)$ & -7887 & $6043(7)$ & $5910(8)$ \\
$b_{22} \mathrm{MHz}$ & $865(7)$ & $935(7)$ & -4198 & $9731(9)$ & $9831(8)$ \\
$b_{40} \mathrm{MHz}$ & $-137(2)$ & $-139(2)$ & 28 & $-213(5)$ & $-208(4)$ \\
$b_{42} \mathrm{MHz}$ & $14(8)$ & $15(9)$ & -977 & $-13(16)$ & $41(18)$ \\
$b_{44} \mathrm{MHz}$ & $645(7)$ & $653(8)$ & -812 & $876(15)$ & $844(16)$ \\
$b_{44} / b_{40}$ & -4.71 & -4.7 & -29 & -4.11 & -4.06 \\
$b_{42} / b_{40}$ & -0.10 & -0.11 & -35 & 0.06 & -0.2 \\
$F(N), \mathrm{MHz}$ & $12(36)$ & $8(25)$ & $40(26)$ & $40(26)$ & $45(26)$
\end{tabular}




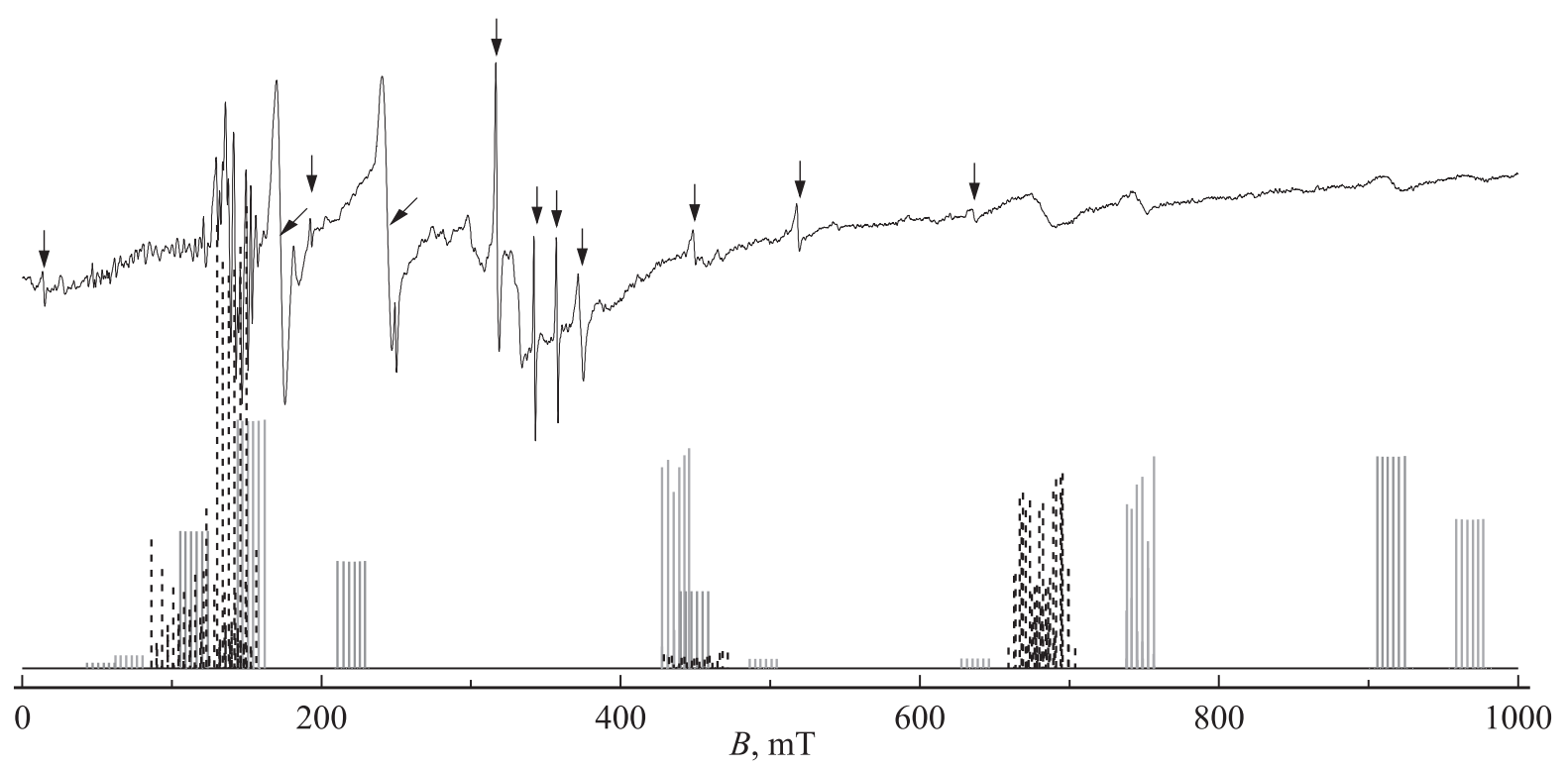

Pис. 2. ЭПР-спектр $\mathrm{Lu}_{3} \mathrm{Al}_{5} \mathrm{O}_{12}: \mathrm{Eu}^{2+}$ при $\mathbf{B} \| \mathbf{C}_{2}$ и 300 К, на частоте $9449 \mathrm{MHz}$. Вертикальные стрелки указывают переходы центров $\mathrm{Gd}^{3+}$, наклонные стрелки - переходы ионов $\mathrm{Mo}^{3+}$. Внизу приведены расчетные положения и интенсивности компонент СТС центров ${ }^{151} \mathrm{Eu}^{2+}$ : пунктирными отрезками показаны компоненты сверхтонкой структуры четырех эквивалентных центров, сплошными - двух одиночных центров.

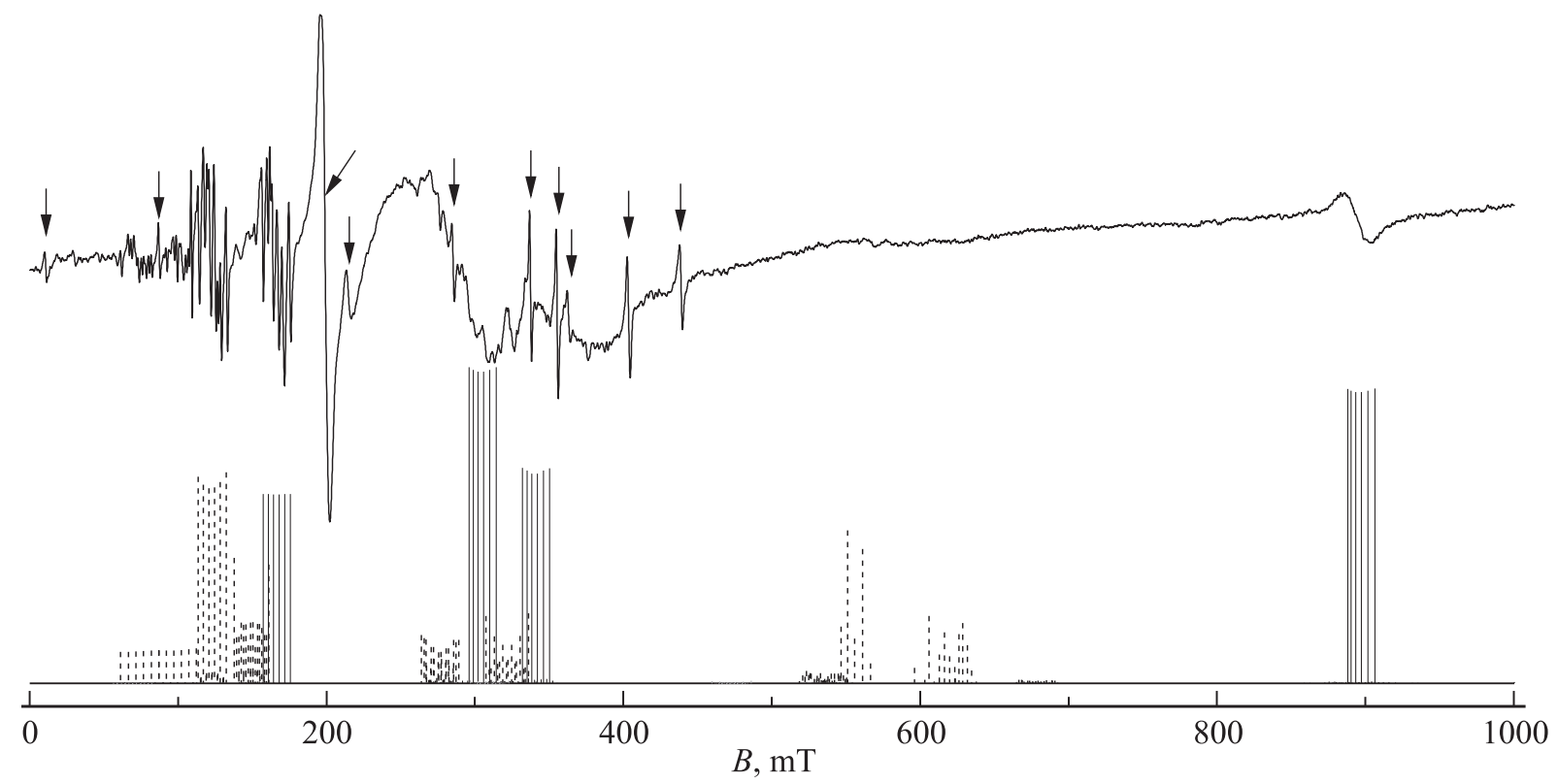

Рис. 3. ЭПР-спектр $\mathrm{Lu}_{3} \mathrm{Al}_{5} \mathrm{O}_{12}: \mathrm{Eu}^{2+}$ при В $\| \mathbf{C}_{4}$ и 300 К на частоте $9449 \mathrm{MHz}$. Вертикальные стрелки указывают переходы центров $\mathrm{Gd}^{3+}$, наклонная стрелка - переход ионов $\mathrm{Mo}^{3+}$. Внизу приведены расчетные положения и интенсивности компонент сверхтонкой структуры центров ${ }^{151} \mathrm{Eu}^{2+}$, пунктирными отрезками показаны компоненты СТС четырех эквивалентных центров, сплошными двух оставшихся эквивалентных центров.

$\mathrm{Eu}^{2+}$ из-за неразрешенной СТС и перекрытия различных переходов.

\section{4. Обсуждение результатов}

Ближайшее кислородное окружение иона $\mathrm{Lu}^{3+}$ в гранате представляет повернутый и скрученный вокруг $C_{4}$ куб, дополнительно искаженный до симметрии $D_{2}$ за счет различия расстояний до ионов кислорода и их полярных углов (рис. 4). При нашем выборе системы координат в процессе обработки эксперимента ось $Z$ может оказаться параллельной (см. разд. 3$)$ либо $C_{2}(1)$, либо $C_{2}(2)$. Следует заметить, что $C_{2}(1)-$ ось $C_{2}$-группы локальной симметрии иона, совпадающая с осью, вокруг которой скручен кислородный куб, тогда как $C_{2}(2)-$ 

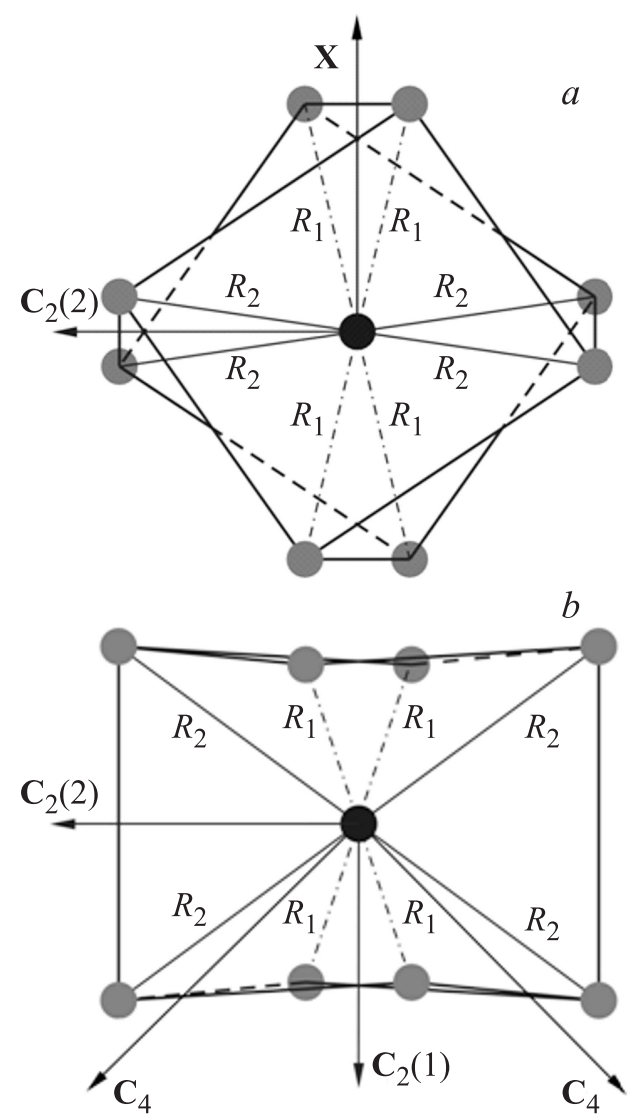

Рис. 4. Ближайшее кислородное окружение позиции иона лютеция в $\mathrm{Lu}_{3} \mathrm{Al}_{5} \mathrm{O}_{12} . a-$ вид вдоль $\mathbf{C}_{2}(1), b-$ вид вдоль $\mathbf{X}\left\|\mathbf{C}_{2}(3)\right\| \mathbf{C}_{4}$ кристалла. Оси, обозначенные как $\mathbf{C}_{4}$, являются осями четвертого порядка кристалла.

ось $C_{2}$ симметрии позиции, близкая к оси второго порядка неискаженного куба. Ось $C_{2}(3)$ симметрии иона $\mathrm{Lu}^{3+}$, как указывалось выше, совпадает с осью четвертого порядка кристалла (рис. 4).

Очевидно, что параметры спинового гамильтониана центров $\mathrm{Gd}^{3+}$ (табл. 1) определены в системе координат $\mathbf{Z} \| \mathbf{C}_{2}(1)$. Об этом свидетельствует отношение параметров четвертого ранга $b_{44} / b_{40}=-4.7$, близкое к величине -5 , а также малая величина $b_{42}$, что характерно для выбора главной магнитной оси параллельно оси четвертого порядка идеального куба.

Для центров $\mathrm{Eu}^{2+}$ в наборе параметров, полученном из обработки экспериментальных данных (табл. 1, первый столбец для европия), отношение $b_{44} / b_{40}=-29$ много больше величины -5 , характерной для кубического инварианта при $\mathbf{Z} \| \mathbf{C}_{4}$. После поворота системы координат на $90^{\circ}$ вокруг оси $X$ (табл. 1) это отношение приобретает величину $b_{44} / b_{40}=-4.1$, близкую к -5 ; кроме того, минимизируется величина $b_{42}$, т.е. ось $Z$ становится параллельной оси $C_{2}(1)$.

Предполагается, что знаки параметров спинового гамильтониана $S$-ионов в $\mathrm{Lu}_{3} \mathrm{Al}_{5} \mathrm{O}_{12}$ (табл. 1) аналогичны знакам в $\mathrm{Y}_{3} \mathrm{Al}_{5} \mathrm{O}_{12}$, полученным для центров $\mathrm{Gd}^{3+}$ авторами [8] и для $\mathrm{Eu}^{2+}$ нами в работе [12]. Абсолют- ный знак параметра $b_{20}$ в [8] определен в результате наблюдения температурного поведения интенсивности различных переходов, а в [12] - из сравнения СТС $\mathrm{Y}_{3} \mathrm{Al}_{5} \mathrm{O}_{12}$, легированного изотопом ${ }^{151} \mathrm{Eu}$, с расчетной структурой при различных и одинаковых знаках $b_{20}$ и константы сверхтонкого взаимодействия $A=-102 \mathrm{MHz}$. Знак $A$ определен в [15-17].

На рис. 2, 3 кроме экспериментальных спектров приведены расчетные положения и интенсивности сверхтонких компонент ${ }^{151} \mathrm{Eu}^{2+}$, полученных в результате диагонализации матрицы энергии 48 порядка. Некоторое несоответствие расчетных и экспериментальных величин интенсивностей обусловлено, в частности, тем, что на рисунках сравниваются интегральные интенсивности компонент СТС с первой производной спектра поглощения. Величина $A \approx-100 \mathrm{MHz}$ оценена по положениям крайних компонент сверхтонкой структуры. В этих расчетах ядерное квадрупольное взаимодействие не учитывалось. Наличие в исследуемом кристалле $\mathrm{Lu}_{3} \mathrm{Al}_{5} \mathrm{O}_{12}$ изотопа ${ }^{153} \mathrm{Eu}^{2+}$ с меньшим сверхтонким взаимодействием и бо́льшим квадрупольным взаимодействием [16], естественно, заметно усложняет вид СТС.

Энергетическая структура основного состояния и резонансные переходы между его подуровнями (рис. 3) центров $\mathrm{Gd}^{3+}$ и $\mathrm{Eu}^{2+}$ (без учета сверхтонкой структуры) при $\mathbf{B} \| \mathbf{C}_{4}$ показаны на рис. 5,6. На рис. 5 для центров $\mathrm{Gd}^{3+}$ показаны только те возможные переходы, расчетные вероятности которых с параметрами из табл. 1 и с учетом взаимной ориентации постоянного и переменного магнитных полей для всех шести магнитно-неэквивалентных центров находятся в диапазоне одного порядка величины. При этом жирными отрезками выделены те переходы, которые наблюдаются в экспериментальном спектре (не перекрыты сигналами $\mathrm{Eu}^{2+}$ и $\left.\mathrm{Mo}^{3+}\right)$ и помечены вертикальными стрелками на рис. 3. Хорошо видно (рис. 5,6), что начальные расщепления центров $\mathrm{Eu}^{2+}$ примерно в 5 раз больше,

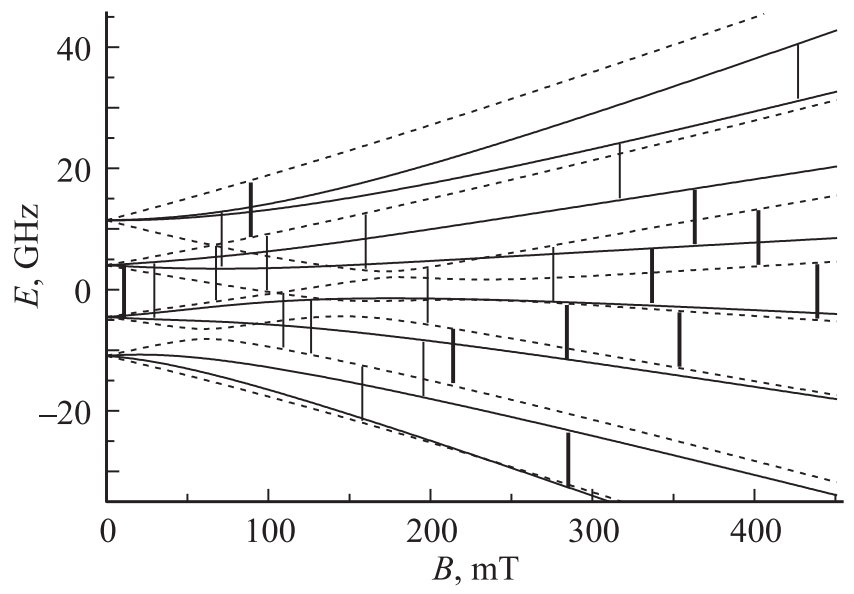

Рис. 5. Расчетные уровни энергии и переходы центров $\mathrm{Gd}^{3+}$ при В $\| \mathbf{C}_{4}$ и 300 К на частоте $9449 \mathrm{MHz}$. Сплошные кривые для двух центров с $\theta=90^{\circ}$, штриховые - для четырех центров с $\theta=45^{\circ}$. Наблюдаемые переходы показаны жирными отрезками. 


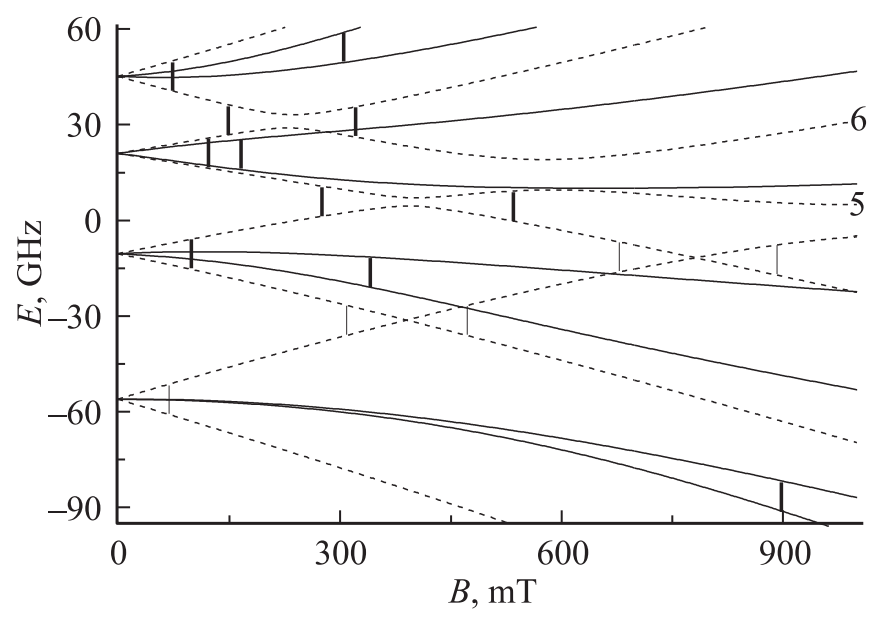

Рис. 6. Уровни энергии и переходы центров $\mathrm{Eu}^{2+}$ при $\mathbf{B} \| \mathbf{C}_{4}$ и 300 К. Сплошные кривые - для двух центров с $\theta=90^{\circ}$, штриховые - для четырех центров с $\theta=45^{\circ}$. Наблюдаемые переходы (рис. 3) показаны жирными отрезками.

чем в случае центров $\mathrm{Gd}^{3+}$, что, в частности, определяет бо́льшую протяженность спектра $\mathrm{Eu}^{2+}$.

На рис. 6 для центров $\mathrm{Eu}^{2+}$ при $\mathbf{B} \| \mathbf{C}_{4}$ приведены все возможные ЭПР-переходы, положения которых рассчитаны в приближении равенства нулю константы сверхтонкого взаимодействия, т.е. для гипотетического четного изотопа. Расчетные вероятности таких переходов находятся в диапазоне трех порядков, регистрируемые сигналы (рис 3) показаны жирными отрезками.

Расчетные положения и интенсивности компонент $\mathrm{CTC}$ центров $\mathrm{Eu}^{2+}$ в этой ориентации магнитного поля были приведены на рис. 3 (для изотопа ${ }^{151} \mathrm{Eu}$ с большей константой сверхтонкого взаимодействия). При сравнении расчетной структуры с экспериментальной, которое, естественно, необходимо проводить с учетом влияния на суммарный спектр СТС изотопа ${ }^{153} \mathrm{Eu}$ ширины компонент на разных электронных переходах и разной кратности групп магнитно-неэквивалентных центров, бросается в глаза разная степень разрешения компонент СТС на низкополевых и высокополевых сигналах (например, в магнитных полях 168 и $900 \mathrm{mT}$ ).

Следуя [12], логично предположить, что наблюдаемая картина обусловлена разным влиянием на эти переходы статического разброса двух самых больших параметров $\left(b_{20}\right.$ и $\left.b_{22}\right)$ спинового гамильтониана. Расчеты с параметрами из табл. 1 для ромбических центров $\mathrm{Eu}^{2+}$ дают $d B_{\text {res }} / d b_{20}=0.002, d B_{\text {res }} / d b_{22}=0.011 \quad\left(B_{\text {res }}-\right.$ peзонансное положение сигнала) для перехода в поле $168 \mathrm{mT}$ и $d B_{\text {res }} / d b_{20}=0.112, d B_{\text {res }} / d b_{22}=0.078$ (все величины в $\mathrm{mT} / \mathrm{MHz}$ ) в поле $900 \mathrm{mT}$.

В пользу приведенного обоснования свидетельствует и ситуация с электронно-ядерными переходами в поле $\sim 600 \mathrm{mT}$ (рис. 3). Энергетический зазор между уровнями энергии 5 и 6 на рис. 6 (штриховые кривые), рассчитанными без учета сверхтонкого взаимодействия, превышает величину используемого в экспериментальной установке кванта СВЧ, поэтому переходов между этими уровнями в поле $\sim 600 \mathrm{mT}$ нет. А расчет с учетом сверхтонкого взаимодействия для изотопа ${ }^{151} \mathrm{Eu}$ показывает, что в этой области магнитных полей уже есть довольно интенсивные компоненты СТС двух электронных переходов (рис. 3), хотя и не между всеми электронно-ядерными подуровнями, а соответствия по интенсивностям в экспериментальном спектре не наблюдается. Объяснением данного факта может являться еще бо́льшая чувствительность этих переходов к изменениям параметров гамильтониана: $d B_{\text {res }} / d b_{20}=0.45 \mathrm{mT} / \mathrm{MHz}$ и $d B_{\text {res }} / d b_{22}=0.45 \mathrm{mT} / \mathrm{MHz}$.

Большие величины параметров $b_{20}$ и $b_{22}$ для центров $\mathrm{Eu}^{2+}$ и соответственно регистрация переходов в трехсан-

Таблица 2. Параметры тонкой структуры ромбических центров $\mathrm{Gd}^{3+}$ и $\mathrm{Eu}^{2+}$ в гранатах при $300 \mathrm{~K}$ (в системе координат $\left.\mathbf{Z}\left\|\mathbf{C}_{2}(1), \mathbf{Y}\right\| \mathbf{C}_{2}(2)\right)$ (знаки параметров в работах [8-10] отличаются от приведенных здесь вследствие использования нами системы координат, повернутой на $90^{\circ}$ вокруг $Z$ )

\begin{tabular}{|c|c|c|c|c|c|}
\hline \multirow{2}{*}{$\begin{array}{c}\text { Параметр, } \\
\text { MHz }\end{array}$} & \multicolumn{3}{|c|}{$\mathrm{Gd}^{3+}$} & \multicolumn{2}{|c|}{$\mathrm{Eu}^{2+}$} \\
\hline & $\mathrm{Y}_{3} \mathrm{Ga}_{5} \mathrm{O}_{12}$ & $\mathrm{Y}_{3} \mathrm{Al}_{5} \mathrm{O}_{12}$ & $\mathrm{Lu}_{3} \mathrm{Al}_{5} \mathrm{O}_{12}{ }^{*}$ & $\begin{array}{l}\mathrm{Y}_{3} \mathrm{Al}_{5} \mathrm{O}_{12} \\
{[11,12]^{* *}}\end{array}$ & $\mathrm{Lu}_{3} \mathrm{Al}_{5} \mathrm{O}_{12}{ }^{*}$ \\
\hline$b_{20}$ & $\begin{array}{l}1331.4[8] \\
1321.0[9]\end{array}$ & $\begin{array}{l}2275.3[10] \\
2275.3[11]\end{array}$ & 1750 & 8987 & 6043 \\
\hline$b_{22}$ & $\begin{array}{l}1978.6[8] \\
1943.5[9]\end{array}$ & $\begin{array}{r}722.3[10] \\
+717.9[11]\end{array}$ & 865 & 8903 & 9731 \\
\hline$b_{40}$ & $\begin{array}{l}-126.5[8] \\
-129.5[9]\end{array}$ & $\begin{array}{l}-130.8[10] \\
-130.1[11]\end{array}$ & -137 & -201 & -213 \\
\hline$b_{42}$ & $\begin{array}{c}0[8] \\
-9[9]\end{array}$ & $\begin{array}{r}-16.5[10] \\
16.9[11]\end{array}$ & 14 & 34.5 & -13 \\
\hline$b_{44}$ & $\begin{array}{l}530.6[8] \\
541.1[9]\end{array}$ & $\begin{array}{l}593.1[10] \\
591.4[11]\end{array}$ & 645 & 914 & 876 \\
\hline
\end{tabular}

* Данные настоящей работы.

** В этих работах используется система координат $\mathbf{Z}\left\|\mathbf{C}_{2}(2), \mathbf{Y}\right\| \mathbf{C}_{2}$ (1). 


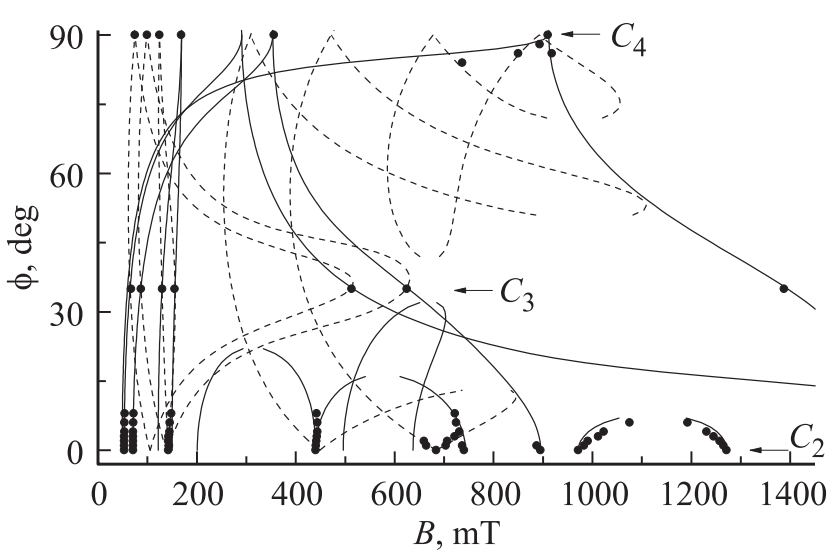

Рис. 7. Угловая зависимость положений переходов центров $\mathrm{Eu}^{2+}$ при вращении магнитного поля в плоскости (110) и $120 \mathrm{~K}$ на частоте $9449 \mathrm{MHz}$. Кривые - расчет, точки - эксперимент. Штриховыми кривыми показаны зависимости центров, спектры которых при В $\| \mathbf{C}_{4}$ и $\mathbf{B} \| \mathbf{C}_{2}$ четырежды вырождены, сплошными - зависимости одиночных при $\mathbf{B} \| \mathbf{C}_{2}$ центров.

тиметровом диапазоне в области промежуточных магнитных полей с неизбежностью должны были привести к очень непростому, трудно регистрируемому ориентационному поведению положений резонансных сигналов. Это и демонстрирует рис. 7, где представлена расчетная угловая зависимость при вращении магнитного поля в плоскости (110). Худшее описание спектра в ориентациях, отличных от $\mathbf{B}\left\|C_{2}, \mathbf{B}\right\| \mathbf{C}_{3}$ и $\mathbf{B} \| \mathbf{C}_{4}$, объясняется выходом магнитного поля из плоскости (110).

Уверенности в адекватности параметров спинового га-

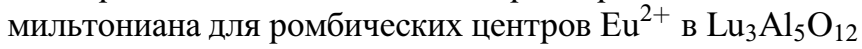
придает тот факт, что, хотя параметры определены по трем ориентациям магнитного поля относительно кристаллографических осей, из-за наличия шести магнитнонеэквивалентных центров они определены в семи ориентациях магнитного поля относительно осей центра (трех при $\mathbf{B} \| \mathbf{C}_{2}$, двух при $\mathbf{B} \| \mathbf{C}_{3}$ и двух при $\left.\mathbf{B} \| \mathbf{C}_{4}\right)$.

В табл. 2 приведены результаты ЭПР-измерений в трех гранатах параметров начальных расщеплений (обозначения [14]) основного состояния редкоземельных $S$-ионов в единой системе координат $\left(\mathbf{Z} \| \mathbf{C}_{2}(1)\right.$, $\left.\mathbf{Y} \| \mathbf{C}_{2}(2)\right)$. Как видно при переходе от $\mathrm{Y}_{3} \mathrm{Al}_{5} \mathrm{O}_{12}$ к $\mathrm{Lu}_{3} \mathrm{Al}_{5} \mathrm{O}_{12}$ параметры спинового гамильтониана второго ранга как $\mathrm{Gd}^{3+}$, так и $\mathrm{Eu}^{2+}$ заметно изменяются (до 1.5 раз), тогда как тензор четвертого ранга остается практически постоянным (изменение $<10 \%$ ).

\section{5. Заключение}

В работе проведено ЭПР-исследование монокристаллов $\mathrm{Lu}_{3} \mathrm{Al}_{5} \mathrm{O}_{12}$, легированных европием и кремнием, измерены спектры ионов $\mathrm{Eu}^{2+}$ и неконтролируемой примеси $\mathrm{Gd}^{3+}$. Определены параметры спинового гамильтониана второго и четвертого рангов ромбических центров, обусловленных ионами $\mathrm{Gd}^{3+}$ и $\mathrm{Eu}^{2+}$, заместившими позиции $\mathrm{Lu}^{3+}$ с локальной симметрией $D_{2}$, в лютецийалюминиевом гранате. Таким образом, количество экспериментальных данных по гранатам для анализа связи параметров тонкой структуры редкоземельных $S$-ионов со структурой их ближайшего окружения в результате проведенных нами исследований заметно увеличилось.

\section{Список литературы}

[1] D.J. Newman. Adv. Phys. 20, 197 (1971).

[2] D.J. Newman, W. Urban. J. Phys. C 5, 3101 (1972).

[3] L.I. Levin. Phys. Status Solidi B 134, 275 (1986).

[4] L.I. Levin, A.D. Gorlov. J. Phys. 4, 1981 (1992).

[5] G. Bacquet, J. Dugas, C. Escribe, J. Michoulier. J. Phys. C 7, 1551 (1974).

[6] J.M. Gaite, G.R. Bulka, N.M. Hasanova, N.M. Nisamutdinov, V.M. Vinokurov. J. Phys. C 19, 2077 (1986).

[7] Н.М. Низамутдинов, Н.М. Хасанова, А.А. Галеев, Г.Р. Булка, В.М. Винокуров, В.А. Аккерман, Г.А. Ермаков. Кристаллография 34, 893 (1989).

[8] L. Rimai, G.A. deMars. J. Appl. Phys. 33, 1254S (1962).

[9] J. Overmeyer, E.A. Giess, M.J. Freiser, B.A. Calhoun. Paramagnetic resonance. Academic Press, N.Y. (1963). V. 1. P. 224.

[10] D.J. Newman, A. Edgar. J. Phys. C 9, 103 (1976).

[11] В.А. Важенин, А.П. Потапов, Г.Р. Асатрян, Ю.А. Успенская, А.Г. Петросян, А.В. Фокин. ФТТ 58, 1573 (2016).

[12] В.А. Важенин, А.П. Потапов, Г.Р. Асатрян, А.Г. Петросян, К.Л. Ованесян, А.В. Фокин, Г.С. Шакуров. ФТТ 58, 2406 (2016).

[13] Э.Г. Шароян, О.С. Торосян, А.Г. Петросян, Э.А. Маркосян. Изв. АН АрмССР. Физика 12, 62 (1977).

[14] С.А. Альтшулер, Б.М. Козырев. Электронный парамагнитный резонанс. Наука, М. (1972). С. 121.

[15] J. Bronstein, V. Volterra. Phys. Rev. 137, A1201 (1965).

[16] J.S.M. Harvey, H. Kiefte. Can. J. Phys. 47, 1505 (1969).

[17] А.Д. Горлов. ФТТ 56, 2115 (2014). 